2017-09

Thermodynamics of an OWC containing real gas

\author{
Medina-Lopez, Encarnacion
}

http://hdl.handle.net/10026.1/17715

10.1016/j.energy.2017.06.164

Energy

Elsevier BV

All content in PEARL is protected by copyright law. Author manuscripts are made available in accordance with publisher policies. Please cite only the published version using the details provided on the item record or document. In the absence of an open licence (e.g. Creative Commons), permissions for further reuse of content should be sought from the publisher or author. 


\title{
Thermodynamics of an OWC containing Real Gas
}

\author{
E. Medina-López ${ }^{\mathrm{a}, \mathrm{b}}$, A. Moñino ${ }^{\mathrm{a}}$, A.G.L. Borthwick ${ }^{\mathrm{b}}$, M. Clavero $^{\mathrm{a}}$ \\ ${ }^{a}$ Andalusian Institute for Earth System Research, Universidad de Granada. Av. del \\ Mediterráneo s/n. 18006, Granada (Spain) \\ ${ }^{b}$ Institute for Energy Systems. School of Engineering. The University of Edinburgh. The \\ King's Buildings, EH9 3JL Edinburgh (United Kingdom)
}

\begin{abstract}
Oscillating Water Column (OWC) devices are usually modelled as simple systems containing ideal, dry air. However, high humidity levels are likely to occur in a prototype device open to the sea, particularly in warm climates such as prevail in the lower latitudes. In this paper, a real gas model is implemented to take into account humidity variations inside an OWC chamber. Using a modified adiabatic index, theoretical expressions are derived for the thermodynamic state variables including enthalpy, entropy and specific heat. The model is validated against experimental data, and shown to provide better agreement than obtained using the ideal gas assumption. By calculating real air flow in an OWC it is shown that the mechanical efficiency reduces and the flow phase alters with respect to the ideal gas case. Accurate prediction of efficiency is essential for the optimal design and management of OWC wave energy converters.
\end{abstract}

Keywords: Oscillating Water Column (OWC), real gas, humidity, Thermodynamics, Adiabatic process, Adiabatic index

\section{Introduction}

Ocean energy offers future opportunities for device developers and stakeholders working in renewable energy technology, and presently has the highest annual growth rate in the sector, Krewitt et.al (2009). To bring forward ocean

5 energy technology, research is being undertaken aimed at field deployment of single prototypes and arrays of wave energy converters (WECs). Of the many pcean energy devices that have been invented, Rosa-Santos et al. (2015), López et al. (22013), the Oscillating Water Column (OWC) is a particularly attractive proposition. The OWC has been the subject of much dedicated research, Falcão (2010), and is one of the few devices to have been tested at full scale under prototype conditions, Cruz (2008). The OWC consists of a partially submerged

Email address: e.medina-lopez@ed.ac.uk (E. Medina-López) 
air chamber, inside which wave action induces air to undergo compressionexpansion cycles thus converting wave energy into pneumatic energy. The OWC is equipped with a power take-off system (PTO), usually consisting of a Wellstype turbine.

Much research attention has focused on the performance of OWCs in delivering power to the grid (see e.g. Medina-López et al. (2016)). Among others, Gato \& Falcão (1984), Gato \& Falcão (1989), Raghunathan (1995), and

20 Sarmento et al. (1990) have carried out detailed studies of the technical behaviour of the Wells turbine when used as a PTO device. Accurate power and efficiency predictions are prerequisites in the design analysis of an OWC converter. According to SI Ocean (2014-II), reliable prediction of energy extraction leads to improved design, better management during service time, 25 increased annual energy production, and lower capital and operating costs. Observed deviations between ideal-gas theory and experimental measurements provide an understanding of the discrepancies between predicted and actual power efficiency estimates for OWC plants currently under development, The Carbon Trust (2005). Predicted efficiency values for wave-pneumatic-electric

30 conversion lie within the $40-70 \%$ range, whereas observed values fall as low as $10 \%$. This is especially relevant to the deployment of devices in less energetic wave climates such as the Mediterranean Sea, Stefanakos et al. (2004).

From a theoretical standpoint, the physics of an OWC is usually described 35 in terms of a classical radiation-diffraction problem, following Evans (1982), Sarmento \& Falcão (1985) and Evans \& Porter (1995). Reviews of the mathematical formulation and various analytical solutions accounting for boundary conditions imposed by either a coastline or a breakwater are given by MartinsRivas \& Mei (2009-I) and Martins-Rivas \& Mei (2009-II).

40

Previous theoretical research on air compression and expansion inside an OWC has assumed the thermodynamic processes to be adiabatic involving ideal gas, without accounting for the prevailing atmospheric conditions. Even when the assumption that the process is adiabatic appears totally justified (see e.g.

${ }_{45}$ Falcão \& Justino (1999) and Sheng et al. (2013)), local temperature and moisture conditions might cause deviation from the polytropic ideal gas model. In fact, air confined inside the chamber and surrounding the OWC is a mixture of dry air and water vapour under ambient sea conditions. The influence of air temperature and moisture on turbine performance is well established for gas so turbines and power plants (see e.g. Yang \& Su (2004), Ibrahim \& Rahman (2010) and Singh \& Kumar (2012)). It is therefore expected that such ambient conditions will influence the performance of the PTO turbine system of an OWC device.

55 Researchers have studied deviations from ideal gas behaviour of the steady flow of an air-water vapour mixture through an OWC chamber model, MedinaLópez et al. (2016). For mass flow and enthalpy to remain conservative over a 
given pressure drop, the temperature change through the turbine could not be obtained theoretically by Medina-López et al. (2016) who assumed an ideal adiabatic polytropic process. A temperature correction had therefore to be applied in accordance with a real gas equation of state based on the virial expansion, Prausnitz (1999). Estimates of pneumatic power using the real gas equation of state were lower than obtained using an ideal gas model. In the general field of thermodynamics however, many researchers have studied the formulation of a

65 model in terms of the virial expansion coefficients for the polytropic process of a real gas (examples include Malic (1955), Pitzer \& Curl (1957), Gel'man \& Smolkin (1966), El-Twaty \& Prausnitz (1981) and|Wisniak (2003)). Moreover, different models have been used to simulate heat transfer, Zhu \& Ye (2010), Liu et al. (2012). Use of virial coefficients in deriving the equations of state for

70 real gases has been predominantly undertaken for applications in the chemical industry, (e.g. Tsonopoulos (1974) and Tsonopoulos \& Heidman (1990)). This approach has provided a useful methodology by which to calculate the system variables of a real gas in terms of deviations from ideal gas values. A preliminary study by Medina-López et al. (2016) has shown that application of 75 real gas formalism to the air-water vapour mixture in an OWC proves to be a valuable tool for the accurate prediction of efficiency.

The aim of this paper is to devise a mathematical formulation for the polytropic process of a real gas that describes the adiabatic compression-expansion

so cycle of a dry air-water vapour mixture in an OWC wave energy converter. The real gas formulation allows us to determine the deviation in PTO efficiency from the expected value under an ideal gas assumption. Experimental data from wind tunnel testing of an OWC chamber will be used to validate the model by comparing values of state variables against predictions by the deduced process equation.

\section{Methodology}

\subsection{Adiabatic process of a real gas: general approach}

We now derive the equation for a polytropic process in a real gas system. Figure 1 illustrates an OWC system from a thermodynamic perspective. The system comprises a chamber, turbine, and external surrounding air. The control volume is independent of the nature of the gas inside the system.

The model is based in the following hypothesis:

- The system is closed. Exchanges of mass and heat in the OWC system take place between the chamber and surrounding air in the vicinity of the device. Provided the surrounding region is sufficiently large, then mass exchange between that region and the atmosphere can be neglected without loss of generality. 


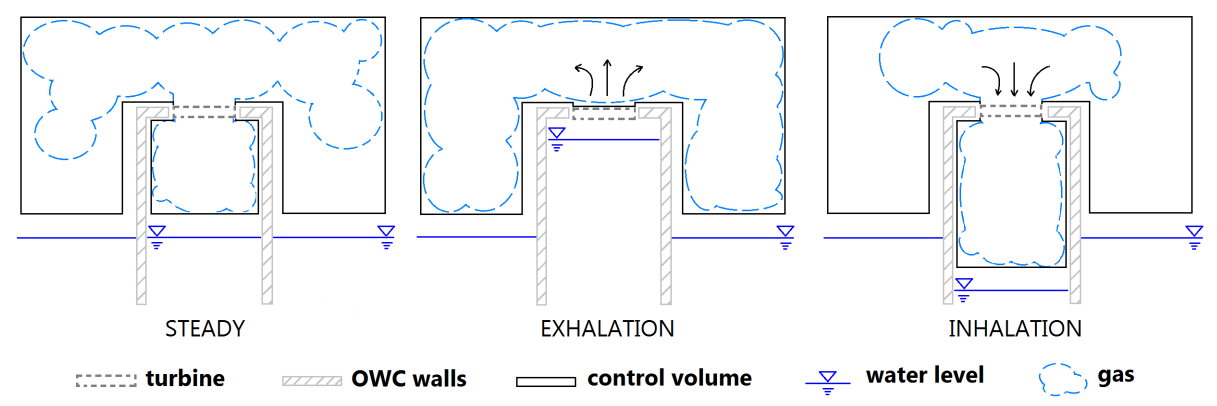

Figure 1: Definition sketch of OWC general scheme, showing control volume.

- The system is isentropic. It is assumed that heat exchange does not occur between the OWC system and either the atmosphere or the surrounding water. This is not a hard restriction. In terms of the exchange process during a compression-expansion cycle, the heat required to address an isothermal process would be greater than that eventually exchanged during the cycle, Falcão \& Justino (1999), leading to an essentially adiabatic process.

- All processes are reversible. If the OWC system is considered similar to gas forced by a frictionless piston (i.e. the free surface inside the chamber) it may be assumed that the OWC system can be returned to the previous state by simply relaxing the compression force for any compression state inside the chamber. Otherwise, the deformation work $\delta L$ exerted on the air chamber cannot be expressed as $-p \delta V$.

In consequence, the starting conditions from which to deduce the equation of a polytropic process of a real gas are the same as those for an ideal gas. The only distinction lies in the equation of state for the real gas. Here, the real gas is described by either the Virial equation of state, or the Kammerling-Onnes expansion, Biel (1997) and Wisniak (2003), which in its Leiden form is given by Prausnitz (1999), as

$$
\frac{p v}{R_{0} T}=1+\frac{B}{v}+\ldots
$$

where $p$ is pressure, $v=V / N$ is the molar volume, with $V$ the volume and $N$ representing the number of moles, $R_{0}=8.31 \mathrm{~J} / \mathrm{mole} \cdot \mathrm{K}$ is the universal gas constant, $B$ is the second virial coefficient (to be determined later), and $T$ is temperature. The real gas equation is defined by analogy to the ideal gas equation, Biel (1997), as

$$
p v=Z R_{0} T,
$$


where $Z$ is the compressibility factor, which models the difference between the real gas and equivalent ideal gas.

\subsection{Polytropic system and adiabatic index $n$ for a real gas}

A thermodynamic process is a succession of different states, progressively altering from an initial equilibrium state to a final one. In this case, the process comprises a compression/exhaust cycle within a polytropic system. The following derivations are based on the standard polytropic formulation (see e.g. Planck (1905), Biel (1997), or Cengel \& Boles (2015)). The polytropic equation for a gas process is:

$$
p v^{n}=\text { constant }
$$

where $n$ is the polytropic index. The General Process Equation (4), Planck (1905), expresses the relationship between $p$ and $v$ for a general process in a

as

$$
\left(\frac{\partial p}{\partial v}\right)_{y}=-\frac{m}{v k_{T}}
$$

where $k_{T}$ is the isothermal compressibility factor, and $m$ is an index defined in terms of the specific heats as:

$$
m=\frac{C_{y}-C_{p}}{C_{y}-C_{v}} .
$$

Specific heats are defined, in general terms, as $C_{y}=T(\partial s / \partial T)_{y}$, where $s$ is the molar entropy, and $y$ depends on the nature of the process. For an isothermal process $y=T$, for an isobaric process $y=p$, and for an adiabatic process $y=s$. Taking the polytropic equation (3) in its differential form, together with the General Process Equation (4) and operating, a general expression for the adiabatic index is obtained:

$$
n=\frac{m}{p k_{T}},
$$

145 where the compressibility factor can be expressed as $k_{T}=-\frac{1}{v}\left(\frac{\partial v}{\partial p}\right)_{T}$. This expression for $n$ is generally applicable to any process in a closed system. Note that if the process is adiabatic for an ideal gas, then $k_{T}=1 / p$, with equation (6) reducing to $n=C_{p} / C_{v}$. In this case, the polytropic index takes a constant value of $n=1.4$ for diatomic molecules, and $n=1.67$ for monoatomic molecules.

150

Next, consider a real gas. Here the real gas equation is obtained as a modification of the ideal gas equation (2). Substituting the partial derivative $\left(\frac{\partial v}{\partial p}\right)_{T}$ into the general expression of polytropic index 6 , and eliminating the 
isothermal compressibility factor $k_{T}$, noting the definitions of the specific heats $C_{p}$ and $C_{v}$, the resulting general expression for $n$ is

$$
n=\frac{m}{1-\frac{p}{Z}\left(\frac{\partial Z}{\partial p}\right)_{T} .}
$$

For an adiabatic process, no heat is transferred to the surrounding universe, and equation (7) then becomes

$$
n=\frac{C_{p} / C_{v}}{1-\frac{p}{Z}\left(\frac{\partial Z}{\partial p}\right)_{T}}
$$

Equation (8) allows us to represent mathematically a real gas process in the continuity equation, applied to an OWC air chamber, given that $p v^{n}=$ const. For the hypothetical case of an ideal gas, then $Z=1$ and equation (8) reduces to $n=C_{p} / C_{v}$, the usual form. The specific heat coefficients, $C_{p}$ and $C_{v}$, refer to a real gas, with expressions that relate to the virial coefficients through thermodynamic formalism. Consequently, equation (8) represents the polytropic index for a general process in a real-gas system.

165 gas:

A simpler form of $n$ can be obtained using the definition of the compressibility factor $Z$ given by Tsonopoulos \& Heidman (1990),

$$
Z=1+\frac{B^{\prime} p_{c} p_{r}}{R_{g} T_{c} T_{r}}=1+\frac{B p_{c} p_{r}}{R_{0} T_{c} T_{r}}
$$

where $B^{\prime}=B / M$ with $M$ the molar weight. Note that $p_{r}=\frac{p}{p_{c}}$ is the reduced pressure, and $T_{r}=\frac{T}{T_{c}}$ is the reduced temperature. Combining 8 and 99 , the following simplified expression is obtained for the adiabatic index $n$ for a real

$$
n=Z \frac{C_{p}}{C_{v}}
$$

The compressibility factor is deduced from the Tsonopoulos-Heidman innovation, and the specific heats for the real gas are expressed as functions of ideal gas values, as (defined in the next section). This form of $n$ is simple, compact 175 and easy to work with.

Figure 2 presents a contour plot of the functional dependence of $n$ on reduced pressure $p_{r}$ and reduced temperature $T_{r}$, expressed by equation (10). Here, the temperature range is from 10 to $25^{\circ} \mathrm{C}$ and the pressure from 0 to $2500 \mathrm{~Pa}$. There is a discernible linear dependence of $n$ on pressure. Taking a reduced pressure ${ }_{180} p_{r}=4.59 \times 10^{-3}$, equivalent to the atmospheric pressure, the adiabatic index $n$ reaches a maximum at 1.33 , decreasing linearly towards 1.3 as the relative 


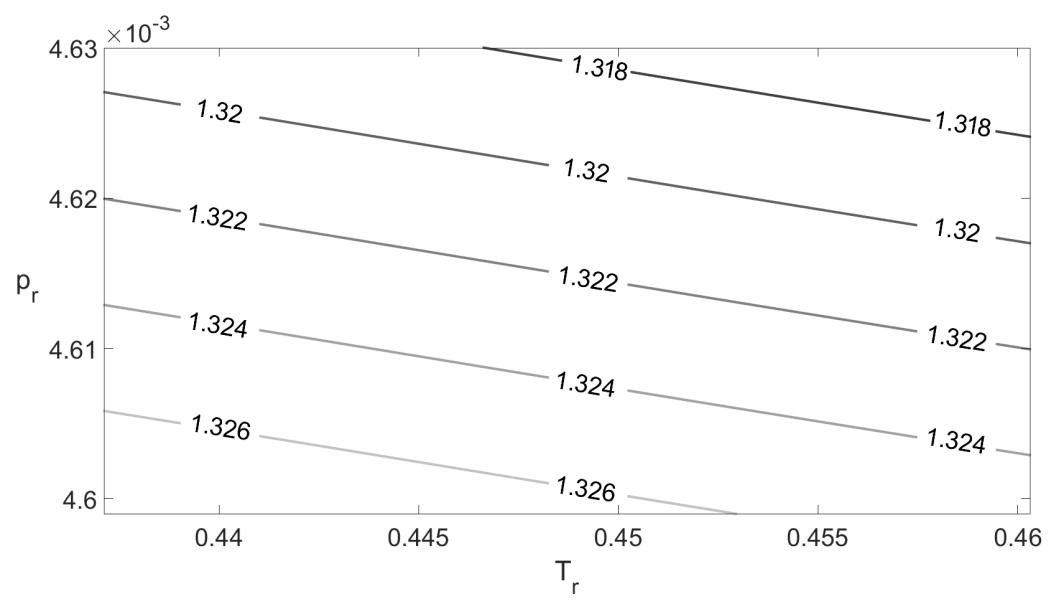

Figure 2: Contour plot of the variation in adiabatic index $n$ with reduced temperature and reduced pressure for a real gas.

\begin{tabular}{lcc}
\hline Constant value for $p v^{n}=$ constant & & \\
\hline$\left(T_{r}, p_{r}\right)$ & Ideal & Real \\
\hline$\left(0.44,4.605 \times 10^{-3}\right)$ & $7.804 \times 10^{4}$ & $7.851 \times 10^{4}$ \\
\hline$\left(0.45,4.620 \times 10^{-3}\right)$ & $7.804 \times 10^{4}$ & $7.851 \times 10^{4}$ \\
\hline$\left(0.46,4.635 \times 10^{-3}\right)$ & $7.804 \times 10^{4}$ & $7.851 \times 10^{4}$ \\
\hline
\end{tabular}

Table 1: Value of the process equation constant obtained using ideal and real gas model.

pressure increases.

Next, consider the constant in equation (3), which is assumed independent of the modified values of $v$ and $p$ for a real gas, and therefore should be insensitive to $n$. Table 1 lists values of the constant for different combinations of relative temperature and pressure. This confirms the initial hypothesis that the adiabatic process remains adiabatic, independent of the value of $n$.

\subsection{Speed of sound in a real gas}

The speed of sound, $C_{s}$, features in the analysis of wave energy extraction devices through the relationship between pressure and density, and affects compressibility. Although the speed of sound is not utilised directly herein, it should be noted that $C_{s}$ must be modified following any change to $n$ in future calculations related to OWC chambers. The speed of sound in an ideal gas 
undergoing an adiabatic process is (Cengel \& Boles (2015)),

$$
C_{s}^{* 2}=\left(\frac{\partial p}{\partial \rho}\right)_{0}=\frac{n p_{0}}{\rho_{0}} .
$$

For an adiabatic process involving a real gas, equation (11) can be modified by applying the real gas equation, $p=\rho Z R_{0} T$, utilizing the definition of the adiabatic index for a real gas. Following the same approach taken previously to $n$, the expression of the speed of sound in a real gas can be simplified using equation $(9)$ to give

$$
C_{s}=\sqrt{\frac{C_{p}}{C_{v}} Z^{2} R_{0} T} .
$$

2.4. Specific heats $\left(C_{p}\right.$ and $\left.C_{v}\right)$, entropy (s), internal energy (u), enthalpy ( $h$ ) and chemical potential $(\mu)$ for a real gas

State variables play a major part in the description of a real gas process. To build up a complete framework of the thermodynamic behaviour of an OWC, expressions for the real gas state variables may be conveniently derived from the ideal gas model. From now on, the ideal gas magnitudes are identified by the superscript index "*". Starting from the definition of $C_{p}$, and applying the Maxwell relations to the definition of the molar entropy $s$, the differential form of s can be obtained. Applying then the virial expansion, (El-Twaty \& Prausnitz (1981)), and operating, the following expression for the molar entropy of a real gas is obtained as, (Biel (1997)),

$$
s(T, p)=s^{*}(T, p)-\frac{d B}{d T} p .
$$

Using the real gas molar entropy, the real gas specific heat at constant pressure is given by:

$$
C_{p} \approx C_{p}^{*}-T \frac{d^{2} B}{d T^{2}} p .
$$

For $C_{v}$ the approximation is not straightforward. Using the differential form of the molar internal energy $u$, and integrating between two pressure states $\left(p_{0}\right.$ and $p$ ), the following expression for the real gas internal energy is obtained:

$$
u(T, p)=u^{*}(T)-T^{2} \frac{R_{0}}{v} \frac{d B}{d T} .
$$

Inserting equation (15) into the definition of $C_{v}$ gives

$$
C_{v} \approx C_{v}^{*}-\frac{R_{0}}{v} \frac{d}{d T}\left(T^{2} \frac{d B}{d T}\right),
$$


where $B$ is the second virial coefficient, defined by Tsonopoulos \& Heidman

220

$(1990)$ as

$$
\frac{B p_{c}}{R_{0} T_{c}}=f_{0}+\omega f_{1}+\chi_{m o l} f_{2} .
$$

The $f$ coefficients, also called temperature correlation functions, are calculated through the Tsonopoulos-Heidman approximation, Tsonopoulos \& Heidman (1990), which expresses the coefficients as functions of the reduced temperature of the real gas, $T_{r}$, given by:

$$
\left\{\begin{array}{l}
f_{0}=0.145-\frac{0.33}{T_{r}}-\frac{0.1385}{T_{r}^{2}}-\frac{0.0121}{T_{r}^{3}}-\frac{0.000607}{T_{r}^{8}} \\
f_{1}=0.0637+\frac{0.331}{T_{r}^{2}}-\frac{0.423}{T_{r}^{3}}-\frac{0.008}{T_{r}^{8}} \\
f_{2}=\frac{0.0297}{T_{r}^{6}}-\frac{0.0229}{T_{r}^{8}} .
\end{array}\right.
$$

225 where $\omega$ is the accentric factor, whose value is almost zero $(\omega \simeq 0)$ for symmetric molecules such as $\mathrm{H}_{2} \mathrm{O}$. In equation (17), $\chi_{m o l}$ is the molar fraction of water vapour in dry air for a given real gas.

In a similar way as for the specific heats, the following expressions for enthalpy and chemical potential of a real gas are obtained:

$$
h(T, p)=h^{*}(T, p)+B p-T \frac{d B}{d T} p,
$$

and

$$
\mu(T, p)=\mu^{*}(T, p)+B p .
$$

The ideal gas values are summarised in Appendix II: List of Symbols.

\subsection{Non dimensional thermodynamic parameters for a real gas}

The thermodynamic parameters derived in Section 2.4 are non-dimensionalized in order to gain a universal perspective of their behaviour under different moisture levels. Using the Buckingham $\Pi$ Theorem, we obtain the following non dimensional numbers:

$$
\begin{aligned}
\Delta \tilde{s} & =\left|\frac{T^{3} R_{0}}{p^{2} B v}\right| \Delta s, \\
\tilde{C_{p}} & =\left|\frac{T}{p B}\right| C_{p}, \\
\tilde{u} & =\left|\frac{T R_{0}}{p^{2} B v}\right| u,
\end{aligned}
$$




$$
\tilde{h}=\left|\frac{T R_{0}}{p^{2} B v}\right| h .
$$

Figures 3(a) and 3(d) show the variations in $\Delta \tilde{s}, \tilde{C}_{p}, \tilde{u}$ and $\tilde{h}$ with $T_{r}$ for three relative humidity conditions, dry air $(R H=0 \%)$, air of medium humidity $(R H=50 \%)$, and saturated air $(R H=100 \%)$. All variables are expressed per mole unit. The reduced temperature range corresponds to that of the experiments conducted by Medina-López et al. (2016). Figure 3(a) indicates that entropy progressively increases with temperature, due to an associated increase in heat in the real gas system. Moreover, saturated gas is more entropic 245 than dry gas, which implies a lower level of energy is available for the saturated gas. This behaviour is corroborated by comparing the entropy-temperature curve with the enthalpy and internal energy counterparts. Energy for saturated gas is lower than for dry gas. And dry conditions are closer to ideal gas. Thus it can be concluded that real gas is more entropic than ideal gas, which translates to lower available energy for real gas. Figure 3(c) shows that the specific heat at constant pressure is consistently lower for a real gas. The heat needed to increase the temperature of a real gas by one degree is lower than for an ideal gas because of intermolecular forces which are only represented by the real gas formulation. In the case of a real gas, when heat is supplied to the system, 255 molecular agitation increases because of repulsive intermolecular forces that promote collisions. This raises the kinetic energy of particles in the system, and so increases the temperature, resulting in less external energy available to increase the system temperature. This leads to lower specific heat for a real gas than for an ideal gas.

The finding can be further explained by considering a scenario where the ideal gas represents a mix of oxygen and hydrogen, whose specific heats at constant pressure are $C_{p}\left(O_{2}\right)=0.9 \mathrm{~J} / \mathrm{K} \cdot g$ and $C_{p}\left(H_{2}\right)=14.3 \mathrm{~J} / \mathrm{K} \cdot \mathrm{g}$. If there is twice the amount of hydrogen than oxygen, then the specific heat of the mixture will be $9.8 \mathrm{~J} / \mathrm{K} \cdot \mathrm{g}$. So, if an equivalent real gas is composed of a molecule of water vapour (formed by independent hydrogen and oxygen molecules present in the ideal gas), the specific heat of the real gas will now be $2 \mathrm{~J} / \mathrm{K} \cdot \mathrm{g}$.

\subsubsection{The real gas non-dimensional number}

A noticeable result is the appearance of a common non-dimensional group, $T R_{0}=p B$, in equations (21) to (24). The group appears as a primary factor in the real gas equation (9). In general terms, the group is defined as

$$
R g=-\frac{T R_{0}}{p B}=\frac{1}{1-Z} .
$$

Note that $R g$ takes the following limits depending on the nature of the gas:

$$
\left\{\begin{array}{l}
R g \rightarrow \infty, \text { as } Z \rightarrow 1(\text { ideal gas }), \\
R g \rightarrow 1, \text { as } Z \rightarrow 0
\end{array}\right.
$$




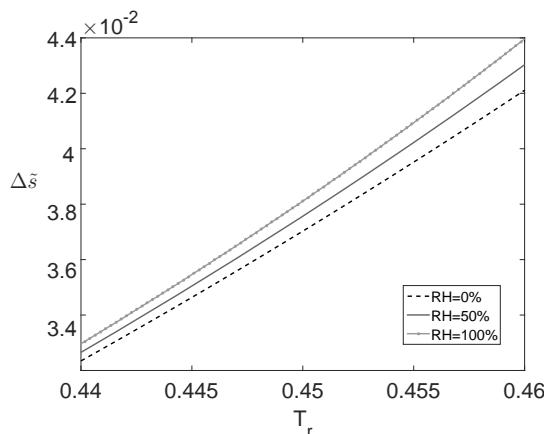

(a)

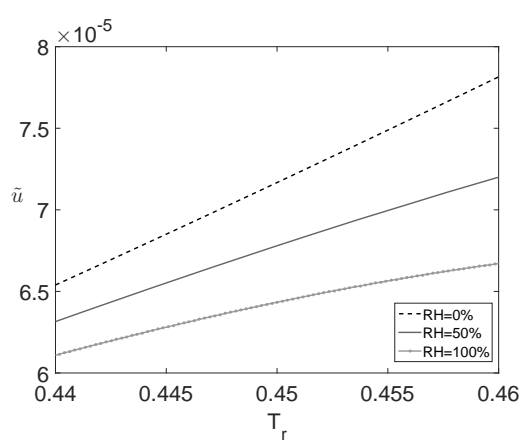

(c)

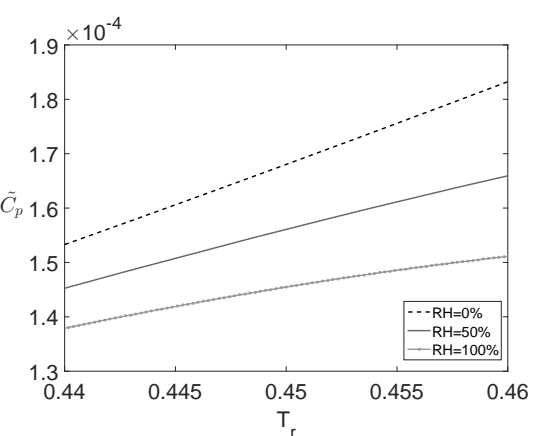

(b)

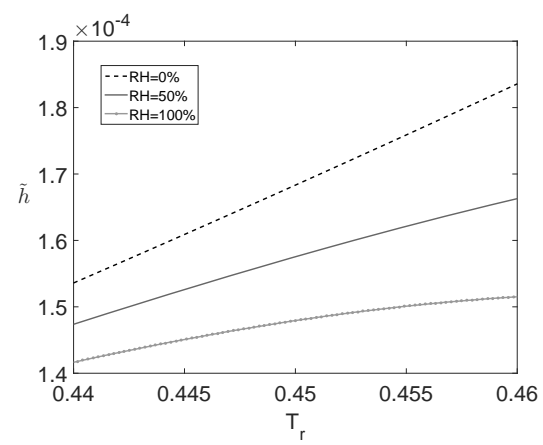

(d)

Figure 3: Variations of (a) non dimensional entropy change, (b) specific heat at constant pressure, (c) internal energy, and (d) enthalpy with reduced temperature for a real gas. 
The closer $R g$ is to 1, the closer the system is to real-gas behaviour. Although the value of $Z=0$ is hypothetical, it provides a useful means by which to obtain a limit that can be used to compare real and ideal gas behaviours. For example, a mixture of dry air and water vapour with a pressure distribution of $[-100,100]$ $\mathrm{kPa}$, relative humidity between $[0 \%-100 \%]$ and temperature range $[15,20]^{\circ} \mathrm{C}$, has values of $Z \in[0.99-0.998]$ and related values of $R g \in[80-500]$.

\section{Experimental validation}

To validate the proposed thermodynamic model, predictions are compared against experimental data obtained by Medina-López et al. (2016) on steady airwater vapour mixture flow through a chamber and turbine. Here, the real gas model is used to predict the change between inner and outer system variables, noted that conservation of mass was not ensured when applying the adiabatic equation for an ideal gas to calculate the temperature at the physical OWC outlet used in the laboratory tests. Herein experimental data are inserted in the formulation of enthalpy to calculate the theoretical outlet velocity, and then used to obtain the mass flow balance in the OWC chamber. Figure 1 indicates the key stages involved in the laboratory tests performed by Medina-López et al. (2016). Experimental data were acquired on flow velocity, air temperature, and pressure at the turbine inlet $\left(U_{i n}, T_{g}, p_{g}\right)$, relative humidity in the chamber $R H$, and pressure at the turbine outlet $p_{\text {out }}$. During the tests, measurements were 295 made of $R H$ at the outlet and $U_{\text {out }}$, but due to non-correspondence between the ideal law applied to the theoretical control volume and the measured variables, a real gas calculation proved necessary in order to confirm the starting hypothesis (real gas under adiabatic process). Commencing from the modified adiabatic index for a real gas, equation $(10)$, values of $Z, C_{p}$ and $C_{v}$ for a real gas are 300 input from the experimental data. Here, the adiabatic index for real gas is obtained from equation (10) as a function of $Z, C_{p}, C_{v}$, and the outlet adiabatic temperature from the real gas calculations. Moreover, a real gas calculation for the outlet velocity is needed. Finally, the real gas density is calculated.

Following Medina-López et al. (2016), the procedure is summarised in Appendix I: Experimental validation procedure Comparison is undertaken between the outlet mass flows obtained by applying the ideal and real gas models to the adiabatic process at the turbine outlet, with respect to the inlet mass flow. At steady-state equilibrium, the inflow and outflow mass flow rates are 310 equal. Table 2 lists the measured inlet and theoretical outlet mass flow rates. There is very good agreement between the predicted mass flow rate obtained using the real gas formulation and the measured mass flow rate. This is not the case for the ideal gas predictions. The present calculations, which use the new approximation for the adiabatic index $n$ for real gas, ensure mass conservation 315

holds within the system. 


\begin{tabular}{lll}
\hline MASS FLOW RATE $\left(\mathrm{kg} / \mathrm{m}^{2} \mathbf{s}\right)$ & $\begin{array}{l}\text { OUT } \\
\text { Adiabatic } \\
\text { Ideal }\end{array}$ & $\begin{array}{l}\text { OUT } \\
\text { Adiabatic } \\
\text { Real }\end{array}$ \\
\hline Dry tests & & \\
\hline 2.077 & 6.198 & 2.054 \\
\hline 5.667 & 13.296 & 5.605 \\
\hline 9.174 & 20.303 & 9.074 \\
\hline 12.559 & 27.109 & 12.423 \\
\hline 15.738 & 33.397 & 15.567 \\
Minimum humidity tests & & \\
\hline 2.242 & 4.848 & 2.218 \\
\hline 3.426 & 9.790 & 3.390 \\
\hline 7.989 & 16.654 & 7.905 \\
\hline 11.146 & 23.469 & 11.030 \\
\hline 14.296 & 30.920 & 14.148 \\
\hline Maximum humidity tests & & \\
\hline 2.502 & 5.488 & 2.475 \\
\hline 5.437 & 10.825 & 5.381 \\
\hline 7.683 & 16.432 & 7.607 \\
\hline 9.926 & 22.672 & 9.828 \\
\hline 12.543 & 29.614 & 12.421 \\
\hline & &
\end{tabular}

Table 2: Mass flow conservation tests, comparing measured inflow rates against predicted outflow rate using ideal and real gas models. 
The ideal gas formulation does not fully guarantee conservation, and so should not be applied when humidity is present. The modified definition leads to a revised adiabatic index that is below the conventional value for dry air of 1.4. Moreover, the fact that mass conservation is achieved through the real gas formulation confirms the starting hypothesis. Here, the real gas hypothesis enables the theoretical model to represent properly actual conditions present in OWC wave energy converters.

\section{Application to OWC formulation}

Using a similar methodology to that of Sheng et al. (2013), who considered an ideal gas, the thermodynamic theory developed herein for real gas is applied to the basic OWC chamber. The subscript " $g$ " is assigned to variables inside the chamber. The mass of air inside the chamber is

$$
m=\rho_{g} V,
$$

where the density $\rho_{g}$ is that of a real gas. The flow rate driven by the water surface movement is defined as

$$
Q_{w}=-\frac{d V}{d t} .
$$

Exhalation occurs when $d m / d t<0$, and inhalation when $d m / d t>0$. This implies that the chamber pressure is greater than atmospheric during exhalation, and the air is decompressed during inhalation. During exhalation, 335 pressurized air is driven out through the PTO system, whereas during inhalation atmospheric air is sucked through the PTO. Hence,

$$
\begin{cases}Q_{p}=-\frac{1}{\rho_{0}} \frac{d m}{d t}, & \text { inhalation, } \\ Q_{p}=-\frac{1}{\rho_{g}} \frac{d m}{d t}, & \text { exhalation. }\end{cases}
$$

In both cases, the power in the chamber is calculated as $P_{w}=p Q_{w}$, taking into account the difference between exhalation and inhalation. The power available to the $\mathrm{PTO}$ is $P_{P T O}=p Q_{p}$.

340

For an adiabatic process involving a real gas, the relationship between density and pressure is given by

$$
\frac{p}{\rho_{g}^{n}}=\text { constant }
$$

Then, linearising:

$$
\rho_{g}=\rho_{0}\left(1+\frac{p}{n p_{0}}\right) .
$$


Note that $n$, the adiabatic index for a real gas, depends on temperature and pressure changes, which vary in time. Differentiating equation (31) and substituting the result into 29 gives,

- Inhalation

$$
Q_{p}=\left(1+\frac{p}{n p_{0}}\right) Q_{w}-\frac{V}{n p_{0}} \frac{d p}{d t}-\frac{V p}{p_{0}} \frac{d(1 / n)}{d t}
$$

- Exhalation

$$
Q_{p}=Q_{w}-\frac{V}{n\left(p_{0}+p\right)} \frac{d p}{d t}-\frac{V p}{p_{0}+p} \frac{d(1 / n)}{d t} .
$$

Equations $(33)$ and $(32)$ are essentially extended versions of the equations presented by Sheng et al. (2013), which take into account the effect on $n$ of

Efficiency is calculated as

$$
\eta=\frac{P_{w}-P_{P T O}}{P_{w}} .
$$

Figure 4 shows the efficiency calculated over a full wave cycle, including inhalation and exhalation stages, for a sinusoidal pressure signal of $100 \mathrm{kPa}$ device. The efficiencies during both exhalation and inhalation stages are lower for a real gas than a corresponding ideal gas. The discrepancy between the curves is less than $1.5 \%$ during inhalation, and reaches about $6 \%$ during exhalation. This is caused by the presence of atmospheric air during inhalation. by the conditions outside the chamber because the air is renewed and the air density can be considered as constant. During the first and third eighths of the cycle, the real gas efficiency curve slightly overlaps that of the ideal gas. This is again related to the presence of real air. While the chamber is filling with fresh 365 air (at the beginning of inhalation), the real gas model takes into account the changes in density, and the process is then similar to that of an ideal gas. So long as the chamber is filled with fresh air (peak), the efficiency starts to vary as mixing occurs. During the second part of the inhalation process, the filling velocity starts to decrease, and the real and ideal gas power estimates converge according to equation $(32)$.

A rough calculation is presented to demonstrate the effect of the real gas formulation on the predicted magnitude and phase of the airflow through the OWC turbine. Air flow in the OWC is calculated for exhalation and inhalation 375 processes driven by harmonic pressure change using equations 32 and 33 for real and ideal gas scenarios. The results are compared to predictions by 


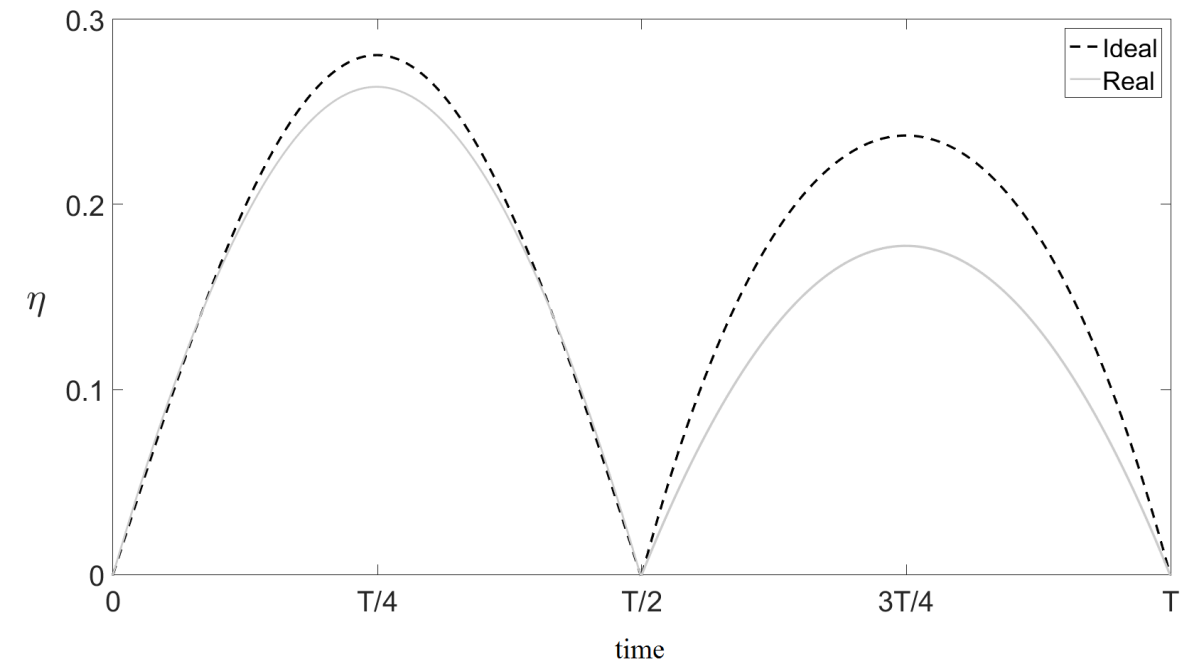

Figure 4: Temporal variations of efficiency of an OWC covering the inhalation (first half of the cycle) and exhalation (second half of the cycle) stages over one oscillation period $T$, for a sinusoidal pressure signal of amplitude $100 \mathrm{kPa}$.

Sheng et al. (2013) for an ideal gas flow. Figure 5 shows the time-dependent phase difference $(\Delta \psi)$ obtained between the real and ideal air flow estimates over a complete pressure cycle. The curve exhibits almost no phase difference in the middle of the cycle because the pressure drop and associated water surface movement are both close to zero, and the ideal and real air flow models give the same results. As the pressure drop increases, the phase difference also increases, exhibiting a peak soon after the start of the cycle. The peak and trough represent the end of the expansion and compression process, and associated minimum and maximum water levels in the chamber. At $t=0, T, 3 T$, etc. the water surface is alternately located at one of its extremes, thus explaining the large difference between phases. Throughout the cycle, the phase gradually alters direction, with expansion and compression ending at opposite symmetric points. Note that if the two processes immediately succeed each other, then the 390 first will start at its maximum, ending at zero, after which the second process starts at zero and ends at its minimum.

\section{Conclusions}

To date most analyses of the thermodynamic behaviour of an oscillating water column have been based on ideal gas theory. This paper has extended the analysis to a real gas by deriving mathematical expressions for the adiabatic index which accounts for a water vapour-dry air mixture. The resulting index 


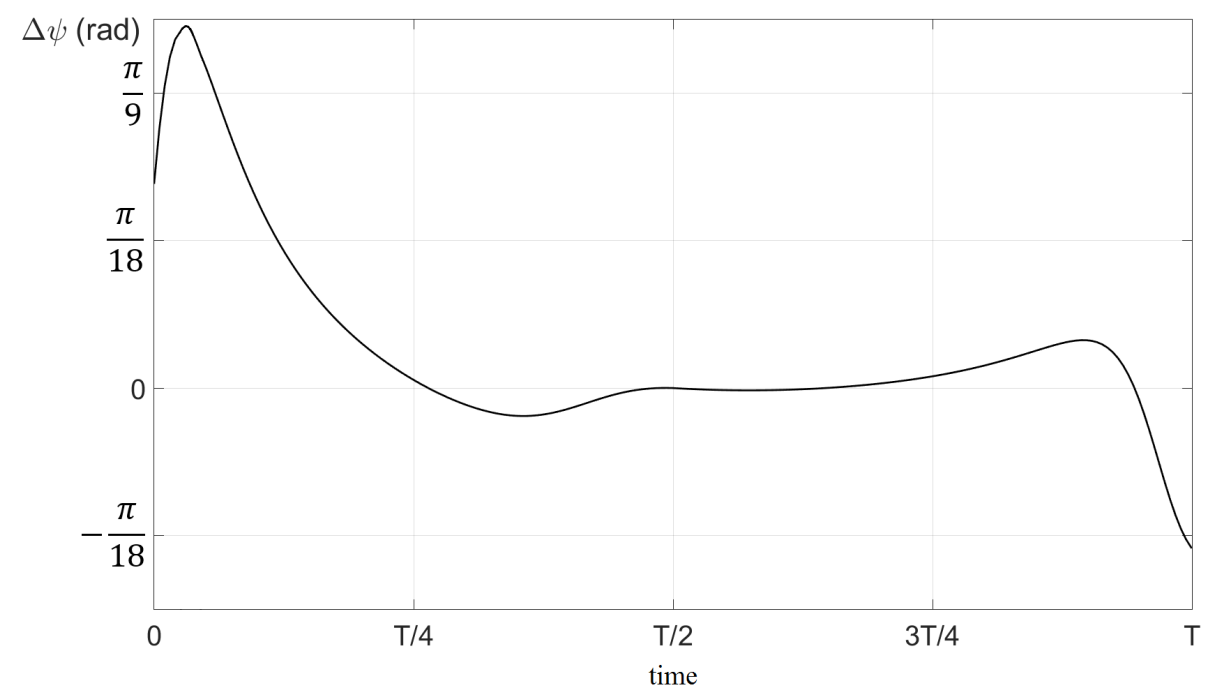

Figure 5: Temporal variation in phase difference between real and ideal air flows for inhalation (first half of the cycle) and exhalation (second half) over a pressure oscillation period (T) in an OWC chamber.

can be readily implemented in a numerical model, and is very straightforward to apply. Modified expressions have been developed for the specific heat coefficients, entropy, internal energy, enthalpy, chemical potential, and speed of sound, all of which apply to a real gas. Unlike ideal gas theory, the real gas theory is found to give excellent agreement with experimental data on mass flow conservation and energy balance through a laboratory-scale OWC. It is found that the adiabatic index depends linearly on temperature and pressure. ${ }_{405}$ The real gas theory has also been applied to the hypothetical OWC device proposed by Sheng et al. (2013), obtaining modified expressions for inhalation and exhalation of the air flow. It is shown that differences between the ideal and real gas models can reach $6 \%$. The real gas model explains part of the losses observed in OWC plants, The Carbon Trust (2005). This study analyses the OWC chamber as an isolated system to understand the effects of the real gas formulation in a simple way. However, the changes introduced by the real gas model in the OWC chamber conditions affect the radiation coefficients as presented by Martins-Rivas \& Mei (2009-I). This will modify the OWC-wave interaction patterns, and further variations are expected between ideal and real gas models. Moreover, it is observed that the accuracy of the real gas model to the actual OWC working conditions is mainly dependent on the adiabatic index $n$, particularly on changes in specific heat at constant pressure. A timedependent specific heat is closer to a precise simulation of the real process. The present analysis should be particularly useful to engineering practitioners 
involved in the design of oscillating water columns, and may have more general application to turbines through which water vapour-dry air mixtures pass.

\section{Acknowledgment}

The first author is recipient of a scholarship from the TALENTIA Fellowship Programme, funded by the Regional Ministry of Economy, Innovation, Science

\section{References}

Abanades J., Greaves D. \& Iglesias G., 2014. Wave Farm Impact on the Beach Profile: a Case Study. Coastal Engineering, Vol. 86, pp. 36-44.

Biel GaYé, J., 1997. Curso sobre el Formalismo y los Métodos de la Termodinámica.. Ed. Reverté, 1997. 400 pp. ISBN 9788429143430.

Cengel Y. A. \& Boles M. A., 2015 Thermodynamics. An engineering approach.. McGraw-Hill Education. 2015. ISBN 978-0-07-339817-4.

Cruz J., 2008. Ocean Wave Energy. Current Status and Future Perspectives. Springer-Verlag. 431 p.p. I.s.B.N. $978-3-540-74894-6$.

Davidson J., Giongi S. \& Ringwood V., 2015. Linear Parametric Hydrodynamic Models for Ocean Wave Energy Converters Identified from Numerical Wave Tank Experiments. OceanEngineering, Vol. 103, pp. 31-39.

EL-Twaty A. I. \& Prausnitz J. M., 1981. Generalized Van der Waals Partition Function for Fluids. Modification to Yield Better Second Virial Coefficients. Fluid Phase Equilibria, 5, pp. 191-197.

Evans D. V., 1982. Wave Power Absorption by Systems of Oscillating Pressure Distributions. Journal of Fluid Mechanics, Vol. 114, pp. 481-499.

Evans D. V. \& Porter R., 1995. Hydrodynamic Characteristics of an Oscillating Water Column Device. Applied Ocean Research, Vol. 17, pp. 155-164.

445 FAlcão A. F. DE O., 2002. Control of an Oscillating Wave Energy Plant for Maximum Energy Production. Applied ocean Research, Vol. 24, pp. 73-82.

Falcão A. F. DE O., 2010. Wave Energy Utilization: A Review of Technologies. Renewable and Sustainable Energy Reviews, Vol. 14, pp. 899-918.

FAlcão A. F. De O. \& Justino P. A. P., 1999. OWC Wave Energy Devices with Air Flow Control. Ocean Engineering, Vol. 26, pp. 1275-1295.

Gato L. M. C. \& Falcão A. F. DE O., 1984 On the Theory of the Wells Turbine. Transactions of the ASME, Vol.106, pp. 628-633.

Gato L. M. C. \& Falcão A. F. De O., 1989 Aerodynamics of the Wells Turbine: Control by Swinging Rotor Blades. International Journal of Mechanical Science, Vol.31 (6), pp. 425-434. 
Gel'man L. I. \& Smolkin Y. V., 1966. On the Calculation of Adiabatic Processes in Real Gases. Journal of Engineering Physics, Vol. 11, $\mathrm{N}^{\mathrm{a}}$ 33, pp. 325-328.

Gkikas G. D. \& Athanassoulis D. A., 2014. Development of a Novel Nonlinear System Identification Scheme for the Pressure Fluctuation inside an Oscillating Water Column Wave Energy Converter Part I: Theoretical Background and Harmonic Excitation Case. Ocean Engineering, Vol. 80, pp. 84-99.

He F. \& Huang Z., 2014. Hydrodynamic Performance of Pile-Supported OWC-Type Structures as Breakwaters: an Experimental Study. Ocean Engineering, Vol. 88, pp. 618-626.

HMRC, 2003. Ocean Energy: Development and Evaluation Protocol. Part I. Wave Power. Marine institute of Ireland.

Ibrahim T. K. \& Rahman M. M., 2010. Effects of Operation Conditions on Performance of Gas Turbine Power Plant. National Conference in Mechanical Engineering Research \& Postgraduate Studies, 3-4 December, Faculty of Mechanical Engineering, UMP Pekan, Kuantan, Pang Malasya, pp. 135-144.

Jalón M. L., Baquerizo A. \& Losada M. A., 2016. Optimization at Different Time Scales for the Design and Management of an Oscillating Water Column System. Energy, Vol. 95, pp. 110-123.

Jefferys E. R., 1984 Simulation of Wave Power Devices. Applied Ocean Research, Vol. 6, $\mathrm{N}^{\mathrm{o}} 1$, pp. 31-39.

Justino P. A. P. \& Falcão A. F. DE O., 1999. Rotational Speed Control of an OWC Wave Power Plant. Journal of Offshore Mechanics and Arctic Engineering, Vol. 121, pp. 65-70.

Konde U., 1991. A Power Take-Off Mechanism for Maximizing the Performance of an Oscillating Water Column Wave Energy Device. Applied Ocean Research, Vol. 13 , pp. $75-81$.

Korde U., 1991. On the Control of Wave Energy Devices in Multi-Frequency Waves. Applied Ocean Research, Vol. 13, pp. 132-144.

Kreysig E., 2006. Advanced Engineering Mathematics, 9th edition. Wiley Editions. I.S.B.N. $0-471-72897-7$.

Krewitt W., Nienhaus K., Klessmann C., Capone C., Stricker E., Graus W., ET AL., 2009. Role and potential of renewable energy and energy efficiency for global energy supply Dessau-Roßlau: Federal Environment Agency (Umweltbundesamt). Dec. Report No.: (UBA-FB) 001323/E.

490 Liu H., Xu K., Zhu T. \& Wenjing Y., 2012 Multiple temperature kinetic model and its applications to micro-scale gas flows. Computers \& Fluids, 67, 115-122.

López. I. \& Iglesias G., 2014. Efficiency of OWC Wave Energy Converters: a Virtual Laboratory. Applied Ocean Research, 44, pp. 63-70.

López. I., Pereiras B., Castro F. \& Iglesias G., 2014. Optimisation of Turbine-Induced Damping for an OWC Wave Energy Converter using a RANSVOF Numerical Model. Applied Energy, 127, pp. 105-114. 
López. I., Pereiras B., Castro F. \& Iglesias G., 2015. Performance of $O W C$ Wave Energy Converters: Influence of Turbine Damping and Tidal Variability. International Journal of Energy Research, 39, 4, pp. 472-483.

López, I., Andreu, J., Ceballos, S., Martínez, I. \& Martínez de Alegría, I., 2013. Review of wave energy technologies and the necessary power-equipment. Renewable and Sustainable Energy Reviews, 27, pp. 413-434.

Lovas S., Mei C. \& LiU Y., 2010. Oscillating Water Column at a Coastal Corner for Wave Power Extraction. Applied Ocean Research, Vol. 32, pp. 267-283.

Malic R., 1955. The Equation of Polytropic Process of Real Gases. Journal of the The Franklin Institute, Vol. 259, issue 3, pp. 235-238.

Martins-Rivas H. \& Mei C. C., 2009. Wave Power Extraction from an Oscillating Water Column at the Tip of a Breakwater. Journal of Fluid Mechanics, Vol. 626, pp. 395-414.

Martins-Rivas H. \& Mei C. C., 2009. Wave Power Extraction from an Oscillating Water Column along a Straight Coast. Ocean Engineering, Vol. 36, pp. 426-433.

Medina-López E., Moñino A., Clavero M., Del Pino C. \& Losada M. A., 2016. Note on a Real Gas Model for OWC Performance. Renewable Energy, Vol. 85, pp. $588-597$.

515 Mendoza E., Silva R., Zanuttigh B., Angelelli E., Andersen T., Martinelli L., NørgaArd J. \& Ruol P., 2014. Beach Response to Wave Energy Converter Farms Acting as Coastal Defence. Coastal Engineering, Vol. 87, pp. 97-111.

Pitzer K. S. \& Curl R. F., 1957. The Volumetric and Thermodynamic Properties of Fluids. III. Empirical Equation for the Second Virial Coefficient. Thermodynamic Properties of Fluids, Vol. 79, pp. 2369-2370.

Planck M., 1905. Treatise on Thermodynamics. Dover Publications Inc., 1905. ISBN-13: 978-0486663715.

Prausnitz J., Lichtenthaler R. \& Gomes de Azevedo E., 1999. Molecular Thermodynamics of Fluid-Phase Equilibria. Prentice-Hall. 864 pp. I.S.B.N. $0-13-$ $977745-8$

Raghunathan S., 1995. The Wells Turbine for Wave Energy Conversion. Prog. Aerospace. Sci., Vol. 31, pp. 335-386.

Rosa-Santos, P., Taveira-Pinto, F., Teixeira, L. \& Ribeiro, J., 2015. CECO wave energy converter: Experimental proof of concept. Journal of Renewable and Sustainable Energy, Vol. 7, pp. 061704.

Sarmento A. J. N. A. \& FAlcÃo A. F. DE O., 1985. Wave Generation by an Oscillating Surface-Pressure and its Application in Wave-Energy Extraction. Journal of Fluid Mechanics, Vol. 150, pp. 467-485.

Sarmento A. J. N. A., Gato L. M. C. \& Falcão A. F. De O., 2003. Turbine535 Controlled Wave Energy Absorption by Oscillating Water Column Devices. Coastal Engineering, Vol. 17, $\mathrm{N}^{\circ}$ 5, pp. 481-497. 
Sheng W., Alcorn R., \& Lewis S., 2013. On Thermodynamics of Primary Energy Conversion of $\mathrm{OWC}$ Wave Energy Converters. Renewable Sustainable Energy, 5, 023105 .

540 SI Ocean Energy Project, 2013. Ocean Energy: State of the Art. Strategic Initiative for Ocean Energy. http://www.si-ocean.eu/en/Home/Home/

SI Ocean Energy Project, 2013. Ocean Energy: Cost of Energy and Cost Reduction Opportunities. Strategic Initiative for Ocean Energy. http://www.siocean.eu/en/Home/Home/

545 SI Ocean Energy Project, 2013. Ocean Energy: Gaps and Barriers. Strategic Initiative for Ocean Energy. http://www.si-ocean.eu/en/Home/Home/

Singh S. \& Kumar R., 2012. Ambient Air Temperature Effect on Power Plant Performance. International Journal of Engineering Science \& Technology, Vol. 4, $\mathrm{N}^{\mathrm{a}}$ 8, pp. 3916-3928.

550 Stefanakos C.N., Athanassoulis G.S.,Cavaleri L, Bertotti L. \& Lefevre J.M., 2004. Wind and Wave Climatology of the Mediterranean Sea. Part II: Wave Statistics. Proceedings of the Fourteenth International Offshore and Polar Engineering Conference, Toulon, France, May 23-28.

Stockmayer W. H. \& Beattie J. A., 1942. The second virial coefficient for gas mixtures.. Journal of Chemical Physics, 10, pp. 473-476. DOI: 10.1063/1.1723750.

Teixeira P., Davyt D., Didier E. \& Ramalhais R., 2013. Numerical Simulation of an Oscillating Water Column Device Using a Code Based on Navier-Stokes Equations. Energy, 61, pp. 513-530.

The Carbon Trust, 2005. Oscillating Water Column Wave Energy Converter Evaluation Report. Marine Energy Challenge.

Tsonopoulos C., 1974. An Empirical Correlations of Second Virial Coefficients. AlChE Journal, Vol. 20, $\mathrm{N}^{\mathrm{o}}$ 2, pp. 263-272.

Tsonopoulos C. \& Heidman J. L., 1990. From the Virial to the Cubic Equation of State. Fluid Phase Equilibria, Vol. 57, pp. 261-276.

565 Wavenet, 2003. Technical Summary Report on the European Thematic Network on Wave Energy. WaveNet. Results from the Work of the European Thematic Network on Wave Energy, ERK5-CT-1999-20001-2000-2003.

WisNiak J., 2003. Eike Kamerlingh-The Virial Equation of State. Indian Journal of Chemical Technology, Vol. 10, pp. 564-572.

570 Wu J., 1990. Are sound waves isothermal or adiabatic?. American Journal of Physics Vol. 58 (7), pp. 694-696.

YAnG W. \& Su M., 2004. Influence of Moist Combustion Gas on Performance of a Sub-Critical Turbine. Energy Conversion \& Management Vol. 46, pp. 821-832.

ZHU T. \& YE W., 2010. Theoretical and numerical studies of noncontinuum gasphase heat conduction in micro/nano devices. Numerical Heat Transfer, Part B: Fundamentals. Vol. 57, pp. 203-226. 


\section{Appendix I: Experimental validation procedure}

The following procedure is followed to validate experimentally the proposed formulation, Medina-López et al. (2016):

1. Calculate the real gas density $\rho_{g}$ from:

$$
\rho_{g}=\rho_{a} \frac{1+r}{1+1.608 r},
$$

where $\rho_{a}$ is the dry air density and $r=\frac{\epsilon e}{p_{g}-e}$ is the absolute humidity. The ratio between dry air and water vapour gas constants, $\epsilon=R_{a} / R_{v}=0.622$. $p_{g}$ is the gas pressure inside the chamber. The vapour pressure $e=R H e_{s}$. Relative humidity $(R H)$ is measured experimentally, and saturated vapour pressure $\left(e_{s}\right)$ can be evaluated from the Clapeyron-Clausius equation, which depends solely on temperature.

2. Calculate the compressibility factor $Z$ using equation (9), where the second term is defined by equation (17) and the $f$ coefficients are calculated through the Tsonopoulos-Heidman approximation, defined by (18).

590

3. Calculate real gas specific heats, $C_{p}$ and $C_{v}$.

4. Calculate the adiabatic index for real gas $n$ from equation 10.

5. Calculate the gas temperature at the outlet as

$$
T_{\text {out }}^{\text {ad,re }}=T_{g}\left(\frac{p_{\text {out }}}{p_{\text {in }}}\right)^{\left(\frac{n-1}{n}\right)},
$$

where $T_{g}$ is the temperature of air in the OWC chamber, and $p_{\text {out }}$ and $p_{\text {in }}$ are the outlet and inlet air pressures, respectively.

6. Calculate the enthalpy at the outlet for a real gas as

$$
H=H^{*}+\delta H p_{\text {out }},
$$

where $H^{*}=C_{p}^{*} T+\frac{1}{2} U^{2}$ is the enthalpy of the ideal gas at the outlet. The deviation from the ideal enthalpy $\delta H$ can be calculated as

$$
\delta H=\frac{R_{g} T_{c}}{p_{c}}\left[\left(f_{0}+\chi_{m} f_{2}\right)-T_{r} \frac{d}{d T_{r}}\left(f_{0}+\chi_{m} f_{2}\right)\right] .
$$

7. Calculate the outlet velocity from the definition of enthalpy expressed as

$$
U_{\text {out }}=\sqrt{2\left(H_{g}-C_{p} T_{\text {out }}-\delta H p_{\text {out }}\right)} .
$$

8. Finally, employ mass conservation between both sides of the turbine to obtain

$$
\rho_{g}^{\text {in }} U_{\text {in }}=\rho_{g}^{\text {out }} U_{\text {out }} .
$$

assuming that the inlet and outlet have the same cross-sectional area. 


\section{Appendix II: List of Symbols}

$600 B$-Second virial coefficient

$B^{\prime}=B / M$ - Second virial coefficient per gas mole

$C_{p}=T\left(\frac{\partial s}{\partial T}\right)_{p}$ - Specific heat for real gas at constant pressure

$C_{p}^{*}=1010 \mathrm{~J} / \mathrm{K} \cdot \mathrm{kg}$-Specific heat for ideal dry air at constant pressure

$\tilde{C}_{p}$-Non dimensional real gas specific heat at constant pressure

${ }_{605} C_{s}^{*}$ - Speed of sound in dry air

$C_{s}$ - Speed of sound in a real gas

$C_{v}=T\left(\frac{\partial s}{\partial T}\right)_{v}$ - Specific heat for real gas at constant volume

$C_{v}^{*}$ - Specific heat for ideal gas at constant volume

$C_{y}=T\left(\frac{\partial S}{\partial T}\right)_{y}$ - Specific heat for constant variable $y$

${ }_{610} e$-Vapour pressure

$e_{s}$ - Saturation vapour pressure

$e_{0}=0.611 \mathrm{kPa}$-Saturation vapour pressure at $273 \mathrm{~K}$

$f_{0}, f_{1}, f_{2}$-Temperature correlation functions

$g$-Acceleration due to gravity

${ }_{615} h^{*}=u^{*}+p V$-Enthalpy per mole unit for ideal gas

$h$-Enthalpy per mole unit for real gas

$\tilde{h}$-Non dimensional enthalpy per mole unit for real gas

$H^{*}$-Enthalpy for ideal gas

$H$-Enthalpy for real gas

${ }_{620} k_{T}=-\frac{1}{v}\left(\frac{\partial v}{\partial p}\right)_{T}$-Isothermal compressibility factor

$L=2.5 \cdot 10^{6} \mathrm{~J} / \mathrm{kg}$-Latent vaporization heat

$m=\frac{C_{y}-C_{p}}{C_{y}-C_{v}}$-Index $m$

$m$-Mass

$M$-Molecular weight

${ }_{625} n$-General polytropic index

$P_{P T O}$ - Power available to the turbine

$P_{w}$-Power in the OWC chamber

$p$-Pressure

$p_{c}$-Critical pressure for water vapour, $220.89 \times 10^{5} \mathrm{~Pa}$

${ }_{630} p_{g}$-Pressure of air-water vapour mixture

$p_{i n}$-Pressure at the turbine inlet

$p_{\text {out }}$-Pressure at the turbine outlet

$p_{r}$-Reduced pressure, $p_{r}=p / p_{c}$

$p_{0}$-Reference thermodynamic pressure

${ }_{635} Q$-Heat

$Q_{w}$ - Flow rate driven by water surface inside the OWC chamber

$Q_{p}$-Air flow rate through the OWC turbine

$r$-Absolute humidity

$R_{a}=286.7 \mathrm{~J} / \mathrm{K} \cdot \mathrm{kg}$-Dry air constant

${ }_{640} \quad R_{g}=R_{0} / M$ - Real gas constant

$R_{v}=461 \mathrm{~J} / \mathrm{K} \cdot \mathrm{kg}$-Water vapour gas constant

$R_{0}=8.31 \mathrm{~J} / \mathrm{K} \cdot \mathrm{mol}$-Universal gas constant 
$R g$ - Real gas non dimensional number

$\mathrm{RH}$-Relative humidity

${ }_{645} s^{*}$-Molar Entropy for the ideal gas

$s$-Molar Entropy for the real gas

$S_{i n}$-Turbine inlet section

$S_{\text {out }}$-Turbine outlet section

$T$-Temperature

${ }_{650} \quad T$-Period

$T_{c}$ - Critical temperature for water vapour, $647 \mathrm{~K}$

$T_{g}$-Temperature at the turbine inlet

$T_{\text {out }}$-Temperature at the turbine outlet

$T_{r}$ - Reduced temperature, $T_{r}=T / T_{c}$

${ }_{655} T_{0}=273 \mathrm{~K}$ - Reference temperature

$t$-Time

$u^{*}=\frac{3}{2} R_{0} T$-Internal energy of a system per mole unit for the ideal gas

$u$-Internal energy of system per mole unit for real gas

$\tilde{u}$-Non dimensional internal energy per mole unit for real gas

$660 U$-Internal energy of system

$U_{i n}$ - Air velocity at turbine inlet

$U_{\text {out }}$ - Air velocity at turbine outlet

$v=V / N$-Volume per mole unit

$V$-Volume

$665 x$-Main horizontal direction

$Z$ - Compressibility factor per gas mole

Greek

$\Delta s^{*}=R_{0} \log \left(\frac{T^{5 / 2}}{p}\right)$-Ideal gas entropy per mole unit variation

$\Delta s$ - Real gas entropy per mole unit variation

$670 \quad \Delta \tilde{s}$-Non dimensional real gas entropy per mole unit variation

$\Delta \psi$-Phase variation between ideal and real air flows

$\delta H$-Deviation from ideal enthalpy

$\eta$-Efficiency

$\gamma=C_{p} / C_{v}=1.4$ - Adiabatic index for ideal gas. Heat capacity ratio for air

${ }_{675} \epsilon=R_{a} / R_{v}$ - Ratio of gas constants for dry air and water vapour

$\chi_{m o l}$-Molar fraction of vapour in dry air

$\mu^{*}$ - Chemical potential of ideal gas

$\mu$-Chemical potential of real gas

$\Psi$-Displacement from equilibrium position

${ }_{680} \rho=N / V-$ Molar density

$\rho_{a}$-Dry air density

$\rho_{g}$ - Real gas density

$\rho_{0}$-Reference air density

$\omega$-Acentric factor

${ }_{685}$ Superscript index

* _ variable for ideal gas 